\title{
Beta/Gamma Oscillations and Event-Related Potentials Indicate Aberrant Multisensory Processing in Schizophrenia
}

\author{
Johanna Balz' ${ }^{1 *}$, Yadira Roa Romero', Julian Keil', Martin Krebber', \\ Michael Niedeggen ${ }^{2}$, Jürgen Gallinat ${ }^{3}$ and Daniel Senkowski ${ }^{1 *}$ \\ ${ }^{1}$ Department of Psychiatry and Psychotherapy, St. Hedwig Hospital, Charité - Universitätsmedizin Berlin, Berlin, Germany, \\ ${ }^{2}$ Department of Education and Psychology, Freie Universität Berlin, Berlin, Germany, ${ }^{3}$ Department of Psychiatry and \\ Psychotherapy, University Medical Center Hamburg-Eppendorf, Hamburg, Germany
}

\section{OPEN ACCESS}

Edited by:

Gregor Thut,

University of Glasgow, UK

Reviewed by:

Valentin Wyart,

École Normale Supérieure, France

Brian A. Coffman,

University of New Mexico, USA

*Correspondence:

Daniel Senkowsk daniel.senkowski@charite.de Johanna Balz

johanna.balz@charite.de

Specialty section:

This article was submitted to

Perception Science,

a section of the journal

Frontiers in Psychology

Received: 27 April 2016 Accepted: 18 November 2016 Published: 06 December 2016

Citation:

Balz J, Roa Romero Y, Keil J, Krebber M, Niedeggen M, Gallinat J and Senkowski D (2016)

Beta/Gamma Oscillations and Event-Related Potentials Indicate

Aberrant Multisensory Processing in Schizophrenia.

Front. Psychol. 7:1896. doi: 10.3389/fpsyg.2016.01896
Recent behavioral and neuroimaging studies have suggested multisensory processing deficits in patients with schizophrenia (SCZ). Thus far, the neural mechanisms underlying these deficits are not well understood. Previous studies with unisensory stimulation have shown altered neural oscillations in SCZ. As such, altered oscillations could contribute to aberrant multisensory processing in this patient group. To test this assumption, we conducted an electroencephalography (EEG) study in 15 SCZ and 15 control participants in whom we examined neural oscillations and event-related potentials (ERPS) in the sound-induced flash illusion (SIFI). In the SIFI multiple auditory stimuli that are presented alongside a single visual stimulus can induce the illusory percept of multiple visual stimuli. In SCZ and control participants we compared ERPs and neural oscillations between trials that induced an illusion and trials that did not induce an illusion. On the behavioral level, SCZ (55.7\%) and control participants (55.4\%) did not significantly differ in illusion rates. The analysis of ERPs revealed diminished amplitudes and altered multisensory processing in SCZ compared to controls around $135 \mathrm{~ms}$ after stimulus onset. Moreover, the analysis of neural oscillations revealed altered 25-35 Hz power after 100 to 150 ms over occipital scalp for SCZ compared to controls. Our findings extend previous observations of aberrant neural oscillations in unisensory perception paradigms. They suggest that altered ERPs and altered occipital beta/gamma band power reflect aberrant multisensory processing in SCZ.

Keywords: crossmodal, sound-induced flash illusion, oscillatory activity, electroencephalography, perception, attention, audiovisual, neural oscillations

\section{INTRODUCTION}

An increasing body of literature suggests that individuals with schizophrenia (SCZ) have deficits in the processing and perception of sensory information (Giersch et al., 2013; Onitsuka et al., 2013; Javitt and Freedman, 2015). Research has stated that primary cognitive disturbances in SCZ cause an inability to sufficiently filter and process sensory information, leading to disconnection of information and disrupted binding (McGhie and Chapman, 1961; Shakow, 1963; Freedman et al., 1991; Vlcek et al., 2014). Furthermore, electrophysiological studies have suggested that aberrant 
neural oscillations play a role for cognitive deficits in SCZ (Andreasen, 2000; Lee et al., 2003; Lisman, 2012; PittmanPolletta et al., 2015; Senkowski and Gallinat, 2015; Uhlhaas and Singer, 2015; Keil et al., 2016). Thus far, sensory processing deficits have been most consistently observed in studies using setups with unisensory stimulation. For instance, one study found impairments in visual temporal order judgments in SCZ (Capa et al., 2014). The study indicated an extended visual simultaneity threshold in patients compared to healthy control participants (HC). Another study investigated perceptual fusion of visual stimuli using the three-flash illusion paradigm (Norton et al., 2008; Chen et al., 2014). In this paradigm two flashes are perceived either as one, two, or three flashes, depending on the inter-stimulus interval between the two visual stimuli. The study by Norton et al. (2008) revealed that the three-flash illusion occurred more often in SCZ than in $\mathrm{HC}$ when the inter-stimulus intervals were longer. When the inter-stimulus interval was shorter, HC reported more illusionary three flashes than SCZ. Thus, there is substantial evidence suggesting altered unisensory stimulus processing in SCZ.

In addition to findings of unisensory processing deficits, there is also some evidence for aberrant multisensory processing in SCZ (Foucher et al., 2007; Stekelenburg et al., 2013; Roa Romero et al., 2016a,b; Stevenson et al., 2016; for a review see Tseng et al., 2015). One study examined simultaneity judgments in unisensory and multisensory stimuli and found that SCZ have a lengthened window of simultaneity for unisensory visual, unisensory auditory, and bisensory audiovisual stimuli (Foucher et al., 2007). Another study investigated multisensory processing of audiovisual video clips in which the onset of the sound was either congruent or incongruent with the visual input (Stekelenburg et al., 2013). Using event-related potentials (ERPs), Stekelenburg et al. (2013) found that SCZ lacked on a reduction of the $\mathrm{N} 1$ component to bisensory compared to unisensory auditory stimuli. Such a reduction, which presumably relates to the fact that the visual input precedes the auditory onset, was observed in healthy individuals. The N1 reduction for auditory stimuli is usually interpreted in terms of a valid crossmodal prediction, e.g., when a visual syllable matches an auditory syllable (van Wassenhove et al., 2005). Thus, the absence of the N1 reduction in bisensory stimulation likely reflects aberrant audiovisual processing in patients. Notably, recent studies have also provided first evidence for altered neural oscillations during multisensory processing in patients (Stone et al., 2014; Roa Romero et al., 2016b). Overall, these findings indicate altered multisensory processing in SCZ.

An established paradigm for examining integrative audiovisual processing is the sound-induced flash illusion (SIFI; Shams et al., 2000). Herein, a single flash that is presented alongside two rapidly repeating tones is either perceived as one flash (i.e., no-illusion) or two flashes (i.e., illusion). Hence, this paradigm allows for the direct comparison of physically identical audiovisual stimuli that are either not integrated (i.e., no-illusion) or integrated (i.e., illusion). Thus far, no electrophysiological study has investigated the SIFI in SCZ.
However, a number of electroencephalography (EEG) and magnetoencephalography (MEG) studies have examined the neural correlates underlying the SIFI in healthy participants (Mishra et al., 2007; Keil A. et al., 2014; Balz et al., 2016). For instance, in one study by Mishra et al. (2007), ERPs and neural oscillations to SIFI stimuli that either induced or did not induce an illusion were compared. The authors calculated the difference between ERPs of both conditions and revealed an early modulation of activity in the visual cortex about $30-60 \mathrm{~ms}$ after the offset of the second auditory stimulus. Subjects with higher illusion rates showed larger amplitudes, which the authors interpreted as individual differences in the neural connectivity underlying multisensory integration. The authors also observed differences between illusion and no-illusion trials that started around $90 \mathrm{~ms}$, i.e., in the time range of the auditory $\mathrm{N} 1$ and the visual $\mathrm{P} 1$, after the onset of the first tone. Moreover, in agreement with other reports (Bhattacharya et al., 2002), the analyses of neural oscillations revealed power differences between illusion and no-illusion trials in neural oscillations ranging from 25 to $35 \mathrm{~Hz}$, comprising an enhancement of beta/gamma power for illusion compared to no-illusion trials. Notably, previous unisensory studies in SCZ have shown dysfunctional oscillatory activity in this frequency range (Gallinat et al., 2004; Uhlhaas et al., 2006; Spencer et al., 2008; Leicht et al., 2010, 2015). Nevertheless, while the neural signatures underlying the SIFI have been well characterized in healthy individuals, it is unknown whether SCZ show alterations in the perception and processing of the SIFI. In the present EEG study we examined perception, ERPs, and neural oscillations during the SIFI in SCZ and HC. In accordance with recent findings of altered multisensory processing, we predicted that SCZ show alterations in the perception and neural processing of the SIFI.

\section{MATERIALS AND METHODS}

\section{Participants}

Twenty-four patients with the DSM-IV diagnosis SCZ were recruited from outpatient units of the Charité Universitätsmedizin Berlin. In addition, 24 age, education, gender, and handedness matched HC participants, who were screened for mental disorders with the German version of the Structural Clinical Interview for DSM-IV-R Non-Patient Edition (SCID), participated in the study. Due to the insufficient number (i.e., <30) of illusion or no-illusion trials following the EEG artifact rejection, data of nine SCZ (mean illusion rate $=71.43 \%)$ and seven $\mathrm{HC}$ (mean illusion rate $=81.53 \%)$ were excluded from further analyses. Participants were excluded due to the following reasons: lack of illusion perception (i.e., $<30$ illusion trials; $N=2 \mathrm{SCZ}, N=1 \mathrm{HC}$ ), lack of no-illusion perception (i.e., $<30$ no-illusion trials; $N=7 \mathrm{SCZ}, N=6$ $\mathrm{HC}$ ). The illusion rates for the excluded SCZ and HC did not significantly differ (Mann-Whitney $U$ test $=20, p=0.223$ ). For the final data analysis, the 15 best matching HC were selected (based on age, education, gender, handedness). All patients met DSM-IV-TR criteria for SCZ. The psychiatric diagnosis was 
assessed by a senior psychiatrist at the recruiting institution. The study was conducted in accordance with the Declaration of Helsinki and approved by the ethics committee of the Charite Universitätsmedizin Berlin. All participants provided written informed consent, had normal hearing, normal or corrected to normal vision, and no neurological disorders, alcohol or substance abuse. A random sample of $40 \%$ of all participants underwent a multi-drug screening and all of those tested had negative results. Severity of symptoms in SCZ was assessed with the Positive and Negative Syndrome Scale (PANSS; Kay et al., 1987). To test cognitive performance, the Brief Assessment of Cognition in Schizophrenia (BACS) was assessed (Keefe et al., 2004). Table 1 provides an overview on demographic data, cognitive performance, and clinical scores of the study participants.

\section{Experimental Design}

The experiment was conducted in a sound-attenuated electrically shielded chamber. Stimuli were presented on a CRT monitor with a background luminance of $21 \mathrm{~cd} / \mathrm{m}^{2}$. Six stimulus combinations were presented: $A_{0} V_{1}, A_{0} V_{2}, A_{1} V_{1}, A_{2} V_{0}, A_{2} V_{1}, A_{2} V_{2}$, where the indexed numbers denote the number of auditory $(\mathrm{A})$ and visual (V) inputs. Participants fixated a central white cross while being presented with stimuli of the SIFI paradigm (Figure 1). The participants' task was to report the number of perceived visual stimuli by pressing a button with the index, middle, or ring finger of their right hand to indicate whether they perceived 0 , 1, or 2 flashes, respectively. Each visual stimulus was presented for $10 \mathrm{~ms}$ and consisted of a white disk subtending $1.6^{\circ}$ with a luminance of $89 \mathrm{~cd} / \mathrm{m}^{2}$. Visual stimuli were presented at $4.1^{\circ}$ centrally below the fixation cross. Each auditory stimulus was presented for $7 \mathrm{~ms}$ and consisted of a $73 \mathrm{~dB}$ (SPL) $1000 \mathrm{~Hz}$ sine wave tone. Auditory stimuli were presented from a central speaker below the screen. Three hundred SIFI trials and 150 trials per control condition were presented in random order in eight blocks.

\section{Analysis of Behavioral Data}

For all stimulus combinations, the numbers of reported zero, one or two flashes were calculated relative to the total number of trials in each condition (Supplementary Figure 1). For the critical $\mathrm{A}_{2} \mathrm{~V}_{1}$ condition, illusion rates were calculated as the percentage of two perceived flashes in relation to the total number of $\mathrm{A}_{2} \mathrm{~V}_{1}$ trials. Within SCZ these values were related to the psychopathology scores (PANSS) by using Pearson correlations. To statistically control for the influence of anti-psychotic medication, medication dosage was converted to chlorpromazine equivalent level (Gardner et al., 2010) and entered as covariate to partial correlation analyses in the patient group. We calculated $t$-tests for each condition to compare the behavioral results between SCZ and HC. To account for multiple comparisons, the statistical outcome was Bonferroni corrected.

TABLE 1 | Demographic data, positive and negative syndromes, and cognitive scores in the study participants.

\begin{tabular}{|c|c|c|c|c|c|c|}
\hline & \multicolumn{2}{|c|}{ Patients } & \multicolumn{2}{|c|}{ Controls } & \multicolumn{2}{|c|}{ Statistics } \\
\hline & Mean & $S D$ & Mean & $S D$ & $t$-values & $p$-values \\
\hline Age (years) & 33.87 & 7.23 & 36.13 & 7.91 & -0.819 & 0.420 \\
\hline Education (years) & 10.93 & 1.44 & 10.87 & 1.81 & 0.112 & 0.912 \\
\hline Illness duration (years) & 9 & 4.8 & - & - & - & - \\
\hline \multirow[t]{2}{*}{ Chlorpromazine equivalent level (daily dosage/mg) } & 398.73 & 167.68 & - & - & - & - \\
\hline & \multicolumn{2}{|c|}{$N$} & \multicolumn{2}{|c|}{$N$} & & \\
\hline Gender (m/f) & \multicolumn{2}{|c|}{$12 / 3$} & \multicolumn{2}{|c|}{$12 / 3$} & - & - \\
\hline Handedness ( $r / l)$ & \multicolumn{2}{|c|}{$13 / 2$} & \multicolumn{2}{|c|}{$13 / 2$} & - & - \\
\hline Antipsychotic Medications & \multicolumn{2}{|c|}{15} & \multicolumn{2}{|c|}{-} & - & - \\
\hline Co-medication* & \multicolumn{2}{|c|}{4} & \multicolumn{2}{|c|}{-} & - & - \\
\hline \multicolumn{7}{|l|}{ BACS } \\
\hline Verbal memory & 42.93 & 11.02 & 44.20 & 9.70 & -0.334 & 0.741 \\
\hline Digit & 18.87 & 3.25 & 20.27 & 3.90 & -1.068 & 0.295 \\
\hline Motor & 65.80 & 14.06 & 72.53 & 9.93 & -1.515 & 0.141 \\
\hline Fluency & 49.13 & 12.08 & 50.47 & 15.05 & -0.268 & 0.791 \\
\hline Symbol coding & 54.67 & 11.84 & 54.73 & 14.80 & -0.014 & 0.989 \\
\hline ToL & 16.87 & 3.14 & 17.40 & 2.26 & -0.534 & 0.597 \\
\hline Total score & 248.27 & 41.65 & 259.60 & 36.89 & -0.789 & 0.437 \\
\hline \multicolumn{7}{|l|}{ PANSS } \\
\hline Negative & 18.47 & 3.16 & - & - & - & - \\
\hline Positive & 17.40 & 2.53 & - & - & - & - \\
\hline General & 38.20 & 3.12 & - & - & - & - \\
\hline Total score & 74.07 & 8.81 & - & - & - & - \\
\hline
\end{tabular}

The table depicts demographic data and BACS scores for SCZ and HC, as well as PANSS scores for SCZ. BACS, Brief Assessment of Cognition in Schizophrenia; PANSS, Positive and Negative Syndrome Scale. *Co-medication of antipsychotics and mood stabilizers. 

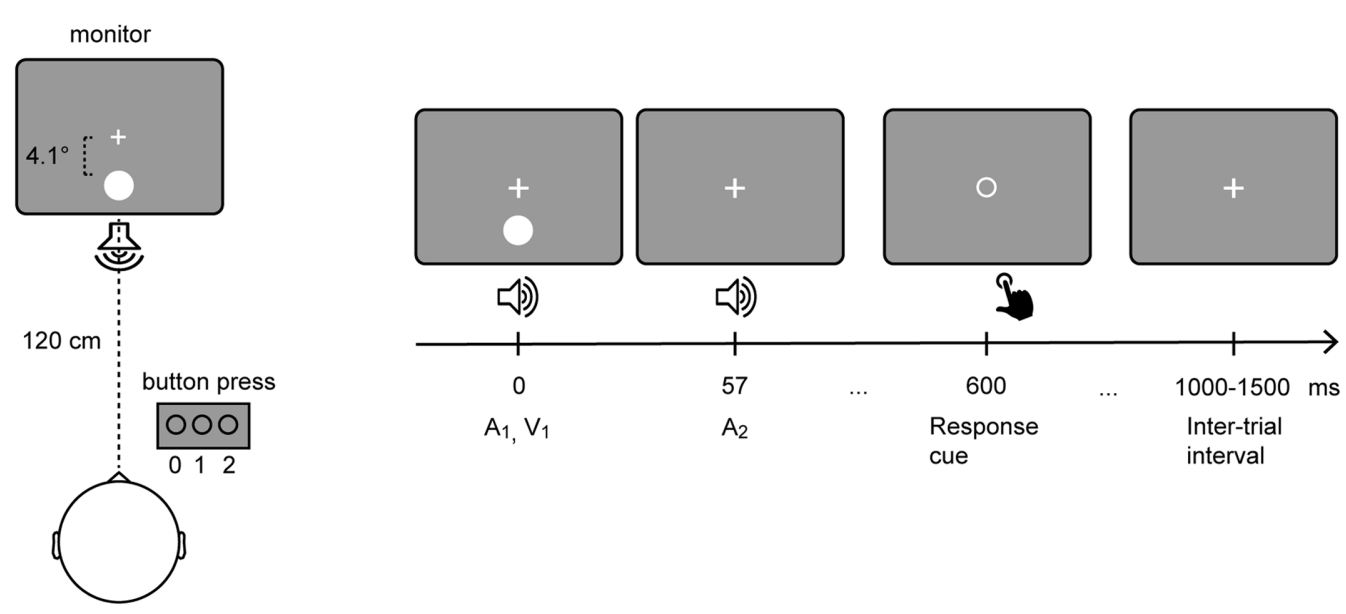

FIGURE 1 | Setup of the sound-induced flash illusion paradigm. Participants fixated a central white cross while being presented with stimuli of the SIFI paradigm (Left). In a critical SIFI trial (i.e., $A_{2} V_{1}$ ) a single flash presented alongside two rapidly repeating tones is either perceived as one or two flashes. (Right) depicts the timeline of the critical SIFI trial. The visual stimulus and the first auditory stimulus are presented simultaneously. The second auditory stimulus is presented $57 \mathrm{~ms}$ after the onset of the first stimulus. Six hundred milliseconds after the onset of the first stimulus, the fixation cross is replaced by a response cue, which comprised an empty circle that is presented in the center of the screen.

\section{Acquisition and Preprocessing of EEG Data}

EEG was recorded using a 128-electrodes active system (EasyCap, Herrsching, Germany), including one horizontal and one vertical EOG electrode to monitor eye movements. Data were recorded against nose reference with a pass band $(0.016-250 \mathrm{~Hz})$ and digitized at a sampling rate of $1000 \mathrm{~Hz}$. Preprocessing and offline data analysis were performed using EEGlab (Delorme and Makeig, 2004), Fieldtrip (Oostenveld et al., 2011), and custom-made Matlab scripts (MathWorks, Natick, MA, USA). In our analyses we followed previously reported procedures (Gross et al., 2013; Gross, 2014; Keil J. et al., 2014). Data were offline high-pass filtered ( $1 \mathrm{~Hz}, \mathrm{FIR})$, low-pass filtered (125 Hz, FIR), and notch-filtered $(49.0-51 \mathrm{~Hz}$, 4th order twopass Butterworth filter). Moreover, data were down-sampled to $500 \mathrm{~Hz}$.

For the data analysis, epochs of $4 \mathrm{~s}(-1$ to $3 \mathrm{~s}$ around the onset of the first auditory stimulus) were extracted and those containing muscular artifacts were rejected by visual inspection. In total, 1050 trials were presented. Trials containing remaining artifacts with amplitudes of $\pm 100 \mu \mathrm{V}$ were rejected automatically. After artifact rejection, we used on average $960.13(S D=53.40)$ trials per individual in the SCZ group for further analyses. In the $\mathrm{HC}$ group we used on average $952.73(S D=62.24)$ trials for further analyses. For the multisensory $\mathrm{A}_{2} \mathrm{~V}_{1}$ trials, we used on average $152.93(S D=67.61)$ illusion trials and $104(S D=54.73)$ no-illusion trials per individual in the SCZ group. In the $\mathrm{HC}$ group we used on average $145.47(S D=56.25)$ illusion trials and 113.53 $(S D=56.08)$ no-illusion trials. For the statistical analysis, the number of $A_{2} V_{1}$ trials was equalized between conditions, i.e., illusion and no-illusion, using the lowest number of available trials in either condition. This was done separately for each individual.
Independent component analyses were conducted to correct for EOG and ECG artifacts (extended runica; Lee et al., 1999). On average, $14.73 \pm 5.06(S D)$ independent components for SCZ and $15.67 \pm 6.17$ independent components for HC were rejected. Next, noisy electrodes were interpolated using spherical interpolation (average SCZ $=1.40 \pm 1.50$ electrodes; $\mathrm{HC}=1.27 \pm 1.58$ electrodes). Finally, the epoched data were rereferenced to common average and the epoch mean was removed from each epoch.

For the ERP analysis of multisensory $\mathrm{A}_{2} \mathrm{~V}_{1}$ trials, data of SCZ and HC were filtered (35 Hz low-pass, 4th order two-pass Butterworth filter), averaged over trials and a baseline correction was performed ( -500 to $0 \mathrm{~ms}$ prior to stimulus onset). ERPs were analyzed in a time interval from 0 to $400 \mathrm{~ms}$ following stimulus onset. Since no prior EEG study has investigated the SIFI in SCZ, we did not have ad hoc hypotheses about the ERP components that could be altered in this patient group. For this reason, we performed non-parametric tests with cluster-based correction for multiple comparisons (Maris and Oostenveld, 2007; Maris, 2012). This was done separately between the different percepts (factor Perception: illusion vs. no-illusion trials), between groups (factor Group: SCZ vs. HC), and for the Perception by Group interaction (i.e., by comparing the differences between illusion vs. no-illusion trials between SCZ and HC). Significant interactions were followed up by post hoc $t$-tests. If an effect was significant, Cohen's $d$ was calculated, as marker of the effect size (Table 2).

To test if there are differences between the processing of multisensory and unisensory stimuli, unisensory visual $\mathrm{A}_{0} \mathrm{~V}_{1}$ and $\mathrm{A}_{0} \mathrm{~V}_{2}$ trials, as well as unisensory auditory $\mathrm{A}_{2} \mathrm{~V}_{0}$ trials were analyzed accordingly. Again, we performed non-parametric tests with cluster-based correction for multiple comparisons between conditions (factor Condition: $A_{0} V_{1}$ vs. $A_{0} V_{2}$ ), between groups (factor Group: SCZ vs. HC), and for the Condition by Group interaction (i.e., by comparing the differences between $\mathrm{A}_{0} \mathrm{~V}_{1}$ 
TABLE 2 | Significant findings of the non-parametric tests.

Multisensory $A_{2} V_{1}$ trials: non-parametric tests with the factors Perception (illusion vs. no-illusion) and Group (SCZ vs. HC)

\begin{tabular}{lcccc}
\hline & \multicolumn{1}{c}{$\begin{array}{c}\text { Time } \\
\text { interval }\end{array}$} & $\begin{array}{c}\boldsymbol{t} \text {-values } \\
\text { (RMS) }\end{array}$ & $\begin{array}{c}\text { Cluster } \\
\boldsymbol{p} \text {-values }\end{array}$ & Cohen's $\boldsymbol{d}$ \\
\cline { 2 - 5 } Perception & $0.11-0.16 \mathrm{~s}$ & 1.766 & - & 0.456 \\
Group & $0.09-0.19 \mathrm{~s}$ & 2.002 & 0.005 & 0.731 \\
Interaction & $0.11-0.16 \mathrm{~s}$ & 2.253 & 0.047 & 0.823 \\
\hline
\end{tabular}

Visual trials: non-parametric tests with the factors Condition $\left(A_{0} V_{1}\right.$ vs. $\mathrm{A}_{0} \mathrm{~V}_{2}$ ) and Group (SCZ vs. HC)

\begin{tabular}{lcccc}
\hline & \multicolumn{1}{c}{$\begin{array}{c}\text { Time } \\
\text { interval }\end{array}$} & $\begin{array}{c}\boldsymbol{t} \text {-values } \\
\text { (RMS) }\end{array}$ & $\begin{array}{c}\text { Cluster } \\
\boldsymbol{p} \text {-values }\end{array}$ & Cohen's $\boldsymbol{d}$ \\
\cline { 2 - 5 } Condition & $0.16-0.27 \mathrm{~s}$ & 1.924 & 0.006 & 0.497 \\
Group & $0.16-0.27 \mathrm{~s}$ & 1.141 & - & 0.417 \\
Interaction & $0.16-0.27 \mathrm{~s}$ & 1.462 & - & 0.534
\end{tabular}

Auditory $\mathrm{A}_{2} \mathrm{~V}_{0}$ trials: non-parametric tests with the factor Group (SCZ vs. HC)

Group n.s.

The table summarizes the results of the non-parametric tests with cluster-based correction for multiple comparisons, which have been conducted for multisensory (upper), visual (middle) and auditory (lower) trials. For each time interval, $t$-values (RMS), significant cluster p-values and Cohen's $d$ are reported.

vs. $A_{0} V_{2}$ trials between SCZ and HC). For the auditory $A_{2} V_{0}$ trials, a similar comparison between groups was conducted. Previous studies on the SIFI have used the additive approach to investigate multisensory interactions in ERPs (Mishra et al., 2008, 2010). In this approach, ERPs to multisensory stimuli are linearly combined with activity from trials in which no stimuli were presented (so called "No-Stim" trials, Busse and Woldorff, 2003; Senkowski et al., 2007). These combined responses, i.e., multisensory AV plus 'No-Stim' ERPs, are then compared with the combined ERPs to the respective unisensory stimuli, e.g., unisensory A plus unisensory $\mathrm{V}$. In the present study, we did not include 'No-Stim' events and did therefore not apply the additive approach.

\section{Time-Frequency Analysis}

In agreement with the finding of a previous study in healthy individuals (Mishra et al., 2007), the analysis of neural oscillations explicitly focused on the examination of beta/gamma band power over the occipital cortex. To this end, time-frequency representations (TFRs) of single trials were calculated for each subject and for each relevant condition (i.e., multisensory: $A_{2} V_{1}$, unisensory: $A_{0} V_{1}, A_{0} V_{2}, A_{2} V_{0}$ ). Morlet Wavelets with a width of seven cycles per frequency were used to calculate spectral estimates at each point of the time-frequency window ranging from -1000 to $1500 \mathrm{~ms}$ (10 $\mathrm{ms}$ steps) in the time domain and 2 to $100 \mathrm{~Hz}$ ( $1 \mathrm{~Hz}$ steps) in the frequency domain. TFRs were baseline-corrected with respect to the interval ranging from -500 to $-100 \mathrm{~ms}$ prior to stimulus onset (absolute baseline). In accordance with Mishra et al. (2007), for the statistical analysis individual mean 25 to $35 \mathrm{~Hz}$ total power in the 100 to $150 \mathrm{~ms}$ interval and individual mean 32 to $40 \mathrm{~Hz}$ total power in the 200 to $240 \mathrm{~ms}$ interval was extracted from a sensor-level ROI consisting of 24 electrodes. The ROI closely matched the 25-35 Hz power topography reported by Mishra et al. (2007). Individual mean power was then submitted to repeated measures $2 \times 2$ ANOVAs with the within-subject factor Perception (illusion vs. no-illusion) and the between-subjects factor Group (SCZ vs. HC). To test if there are differences between the processing of multisensory and unisensory stimuli, an analogous analysis was carried out for unisensory visual stimuli with the within-subject factor Condition $\left(A_{0} V_{1}\right.$ vs. $\mathrm{A}_{0} \mathrm{~V}_{2}$ ) and the between-subjects factor Group (SCZ vs. HC). Significant main effects or interactions were followed up by post hoc $t$-tests. Finally, for the unisensory auditory condition (i.e., $\mathrm{A}_{2} \mathrm{~V}_{0}$ ) a $t$-test was used to test the main effect of Group.

\section{RESULTS}

\section{Behavioral Data}

Schizophrenia reported perceiving two flashes in $55.7 \%$ of the critical $\mathrm{A}_{2} \mathrm{~V}_{1}$ trials, $\mathrm{HC}$ in $55.4 \%$ of the $\mathrm{A}_{2} \mathrm{~V}_{1}$ trials. The illusion rates did not significantly differ between SCZ and HC $[t(28)=0.028, p=0.978]$. Moreover, the behavioral analyses for the other stimulus types revealed that participants correctly reported the number of presented visual and auditory inputs (Supplementary Figure 1). Furthermore, the comparison of the percepts in the control conditions did not reveal any group differences (Supplementary Figure 1). The partial correlation analyses between SIFI illusion rates and clinical symptoms (i.e., PANSS subscale scores), in which medication dose in SCZ served as control variable, were not significant (all $p$-values $>0.2$ ).

\section{Event-Related Potentials}

The non-parametric dependent samples test for multisensory $\mathrm{A}_{2} \mathrm{~V}_{1}$ trials, which was conducted for all electrodes, revealed no significant differences between illusion and no-illusion $A_{2} V_{1}$ trials (Figure 2A). This observation was somewhat surprising and motivated us to conduct an exploratory running nonparametric dependent samples test to examine whether there are ERP differences between illusion and no-illusion $\mathrm{A}_{2} \mathrm{~V}_{1}$ trials in HC only, as previously reported (Mishra et al., 2007). We found significant differences between illusion and no-illusion trials in the 102 to $172 \mathrm{~ms}$ and 314 to $384 \mathrm{~ms}$ intervals, which indicate perception-related modulations of the visual N1 and P3 components specifically in healthy individuals (Supplementary Figure 2). The non-parametric independent samples test between SCZ and HC revealed significant group differences between 88 and $186 \mathrm{~ms}$. More negative amplitudes in HC compared to SCZ were found at a central cluster comprising of 69 electrodes (Figure 2B; Table 2, upper panel). Notably, the nonparametric independent samples test between the Perception differences for SCZ and HC revealed a significant Perception by Group interaction (Figure 2C). The amplitude differences between illusion and no-illusion trials were significantly larger 
A

\section{ERP EFFECTS FOR MULTISENSORY $A_{2} V_{1}$ TRIALS}

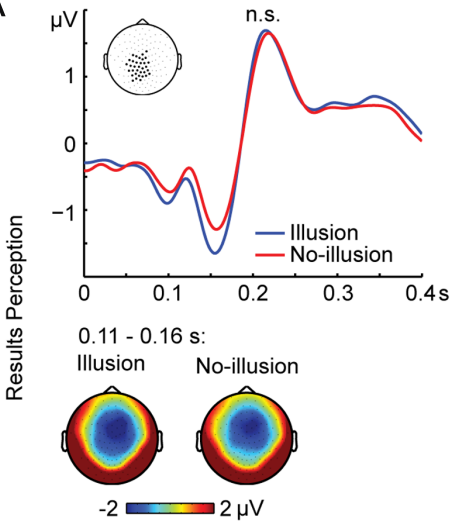

B

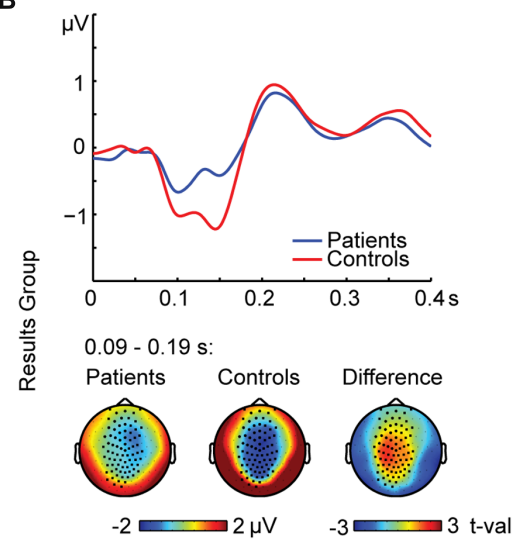

C

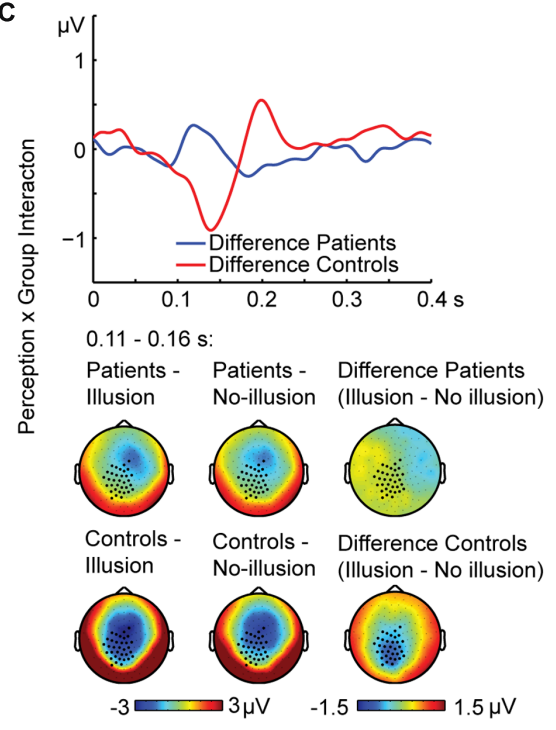

FIGURE 2 | ERP effects of multisensory $\mathbf{A}_{\mathbf{2}} \mathbf{V}_{\mathbf{1}}$ trials. Outcome of the non-parametric tests with cluster-based correction for multiple comparisons between Perceptions (illusion vs. no-illusion), between Groups (SCZ vs. HC), and for the Perception differences between Groups (i.e., the Perception by Group interaction). The upper planes in panels (A-C) illustrate the ERP results for Perception (A), the main effect of Group (B), and the Perception by Group interaction (C). The lower planes depict topographic maps for the observed results with highlighted significant cluster electrodes. Time-point 0 indicates the onset of the first auditory and visual stimulus. for HC than for SCZ, especially between 106 and $164 \mathrm{~ms}$. The interaction patterns were most robust at a cluster comprising of 34 central to posterior electrodes. Follow-up post hoc $t$-tests revealed no differences between illusion compared to noillusion trials in SCZ $[t(14)=0.980, p=0.344]$. However, there were significant differences between illusion compared to no-illusion trials in $\mathrm{HC}[t(14)=-5.220, p=0.000]$. Further testing revealed group differences in the 106 to $164 \mathrm{~ms}$ interval for illusion trials $[t(28)=3.796, p=0.001]$, as well as for no-illusion trials $[t(28)=2.131, p=0.042]$. The ERP amplitudes for both percepts were lower in SCZ compared to HC.

To further examine the effect of visual stimulation alone we conducted non-parametric tests for the unisensory visual conditions (i.e., $A_{0} V_{1}$ vs. $A_{0} V_{2}$ ). For the interval between 164 and $268 \mathrm{~ms}$ we found significant differences between conditions (Figure 3A; Table 2, middle panel). The amplitudes in the $\mathrm{A}_{0} \mathrm{~V}_{2}$ trials compared to $\mathrm{A}_{0} \mathrm{~V}_{1}$ trials were more negative at a cluster comprising of 46 central and posterior electrodes. No main effect of Group or Perception by Group interaction was found (Figures 3B,C). Finally, we compared ERPs to unisensory auditory trials (i.e., $A_{2} V_{0}$ ) between groups. This analysis revealed no significant group differences (Supplementary Figure 3).

\section{Time-Frequency Analysis}

The analysis of neural oscillations focused on occipital 25$35 \mathrm{~Hz}$ power between 100 and $150 \mathrm{~ms}$ and occipital $32-40 \mathrm{~Hz}$ power between 200 and $240 \mathrm{~ms}$. These time-frequency windows were selected based on findings of multisensory interactions in the SIFI obtained by Mishra et al. (2007). The repeated measures $2 \times 2$ ANOVA for multisensory $\mathrm{A}_{2} \mathrm{~V}_{1}$ trials in the 100 to $150 \mathrm{~ms}$ time-frequency window revealed no main effect of Perception $[F(1,28)=0.813, p=0.375]$ or Group $[F(1,28)=1.133, p=0.296]$. However, a significant Perception by Group interaction for the early time-frequency window between 100 and $150 \mathrm{~ms}$ was found $[F(1,28)=4.940, p=0.035$; Figure 4A]. Follow-up $t$-tests revealed significantly larger 25$35 \mathrm{~Hz}$ power in illusion compared to no-illusion trials in $\mathrm{HC}$ $[t(14)=2.502, p=0.025]$, but no power differences between illusion and no-illusion trials in SCZ [ $t(14)=-0.845, p=0.412]$. The analysis of the $32-40 \mathrm{~Hz}$ power in the 200 to $240 \mathrm{~ms}$ time-frequency window did not reveal any significant effects. Analogous to multisensory trials, we calculated power analyses for unisensory visual trials (i.e., $\mathrm{A}_{0} \mathrm{~V}_{1}$ vs. $\mathrm{A}_{0} \mathrm{~V}_{2}$; Figure $4 \mathrm{~B}$ ). The analyses of the 100-150 ms time-frequency window revealed a significant main effect of Condition $[F(1,28)=6.903, p=0.014]$, due to higher power in $A_{0} V_{2}$ compared to $A_{0} V_{1}$ trials. No main effect of Group $[F(1,28)=1.834, p=0.187]$ or Condition by Group interaction $[F(1,28)=1.418, p=0.244]$ was found. The analyses of the 200-240 ms time-frequency window also revealed a significant main effect of Condition $[F(1,28)=4.521$, $p=0.042]$, due to higher power in $\mathrm{A}_{0} \mathrm{~V}_{2}$ compared to $\mathrm{A}_{0} \mathrm{~V}_{1}$ trials. No main effect of Group $[F(1,28)=2.926, p=0.098]$, or Condition by Group interaction $[F(1,28)=0.034, p=0.856]$ was found. Finally, we compared $25-35 \mathrm{~Hz}$ power in unisensory auditory stimuli (i.e., $\mathrm{A}_{2} \mathrm{~V}_{0}$; Supplementary Figure 4) between groups. This analysis did not reveal any significant differences 


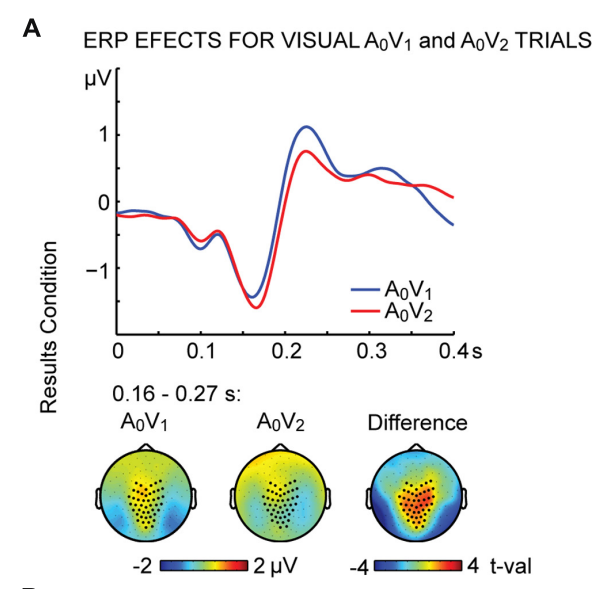

B

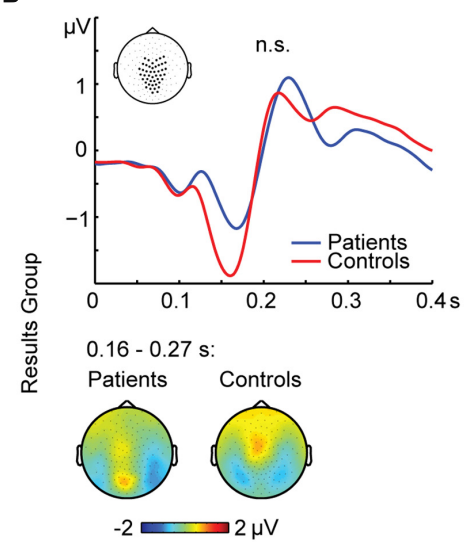

C

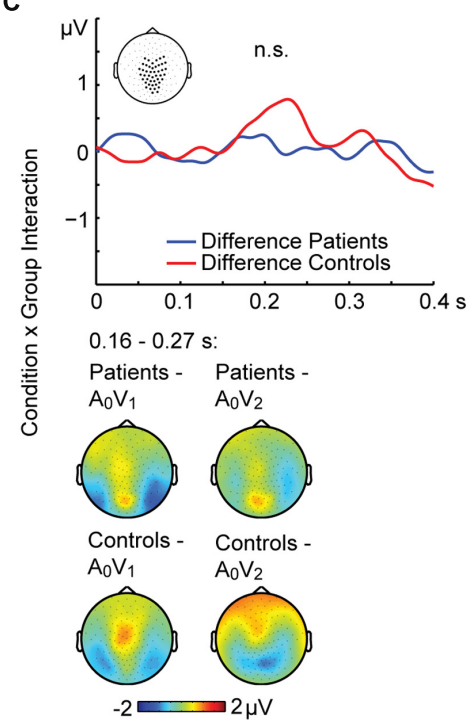

FIGURE 3 | ERP effects of visual $\mathbf{A}_{0} \mathbf{V}_{\mathbf{1}}$ and $\mathbf{A}_{0} \mathbf{V}_{\mathbf{2}}$ trials. Outcome of the non-parametric tests with cluster-based correction for multiple comparisons between Conditions ( $A_{0} \vee_{1}$ vs. $A_{0} V_{2}$ ) between Groups ( $S C Z$ vs. $H C$ ), and for the Condition differences between Groups (i.e., the Condition by Group interaction). The upper planes in panels (A-C) illustrate significant main effects of Condition (A), the ERP results for Group (B) and the Condition by Group interaction (C). The lower planes depict topographic maps for the observed effects with highlighted significant cluster electrodes. Time-point 0 indicates the onset of the first visual stimulus. between SCZ and HC $[t(28)=1.240, p=0.225$ for the $100-$ $150 \mathrm{~ms}$ time-frequency window; $t(28)=1.900, p=0.068$ for the 200-240 ms time-frequency window]. For exploratory purposes we also analyzed alpha-band power between 100 and $300 \mathrm{~ms}$ in the frequency range of $8-13 \mathrm{~Hz}$. The $2 \times 2$ ANOVA did not reveal any significant effects.

\section{DISCUSSION}

In this study, we examined multisensory integration in SCZ and HC using the SIFI paradigm. Our study revealed several interesting findings. Contrary to our hypothesis, on the behavioral level, SCZ and HC did not significantly differ in the perception rates of the SIFI. However, SCZ compared to HC showed reduced amplitudes and diminished ERP differences between illusion and no-illusion trials. Moreover, SCZ lacked an early enhancement of $25-35 \mathrm{~Hz}$ total power for illusion compared to no-illusion SIFI trials, which was observed in $\mathrm{HC}$.

\section{Sound-Induced Flash Illusion Rates Are Comparable between Patients and Healthy Control Participants}

On average, both SCZ and HC perceived the SIFI in about $55 \%$ of the critical $\mathrm{A}_{2} \mathrm{~V}_{1}$ trials. Additionally, there were no perceptual differences between SCZ and $\mathrm{HC}$ in the control conditions. In these conditions, both SCZ and $\mathrm{HC}$ reported the correct numbers of visual stimuli in $73-95 \%$ of trials, indicating that both groups maintained attention throughout the experiment. Hence, the behavioral data suggest no substantial perceptual alterations in SCZ. Given that previous studies have reported aberrant multisensory processing in SCZ (de Boer-Schellekens et al., 2014), the lack of perceptual alterations in the current study is somewhat surprising. In unisensory processing, aberrant neural processing often coincides with altered perception, which is reflected in behavioral deficits (Senkowski and Gallinat, 2015; Uhlhaas and Singer, 2015). Therefore, we expected that differences in multisensory processing in the SIFI would affect the perceptual outcome between the groups. However, it is possible that SCZ show different multisensory processing patterns compared to HC, which still lead to the same perceptual outcome. These different multisensory processing patters might be indicative of compensatory mechanisms, altered signal fidelity or unspecific stimulus processing deficits.

Nevertheless, other studies did also not find differences in the perception of multisensory stimuli between SCZ and HC (Surguladze et al., 2001; Pearl et al., 2009; Martin et al., 2013; Wynn et al., 2014; Roa Romero et al., 2016a). For example, examining the McGurk illusion, Martin et al. (2013), as well as Roa Romero et al. (2016a) observed comparable illusion rates in SCZ and HC. Together with the present findings, these studies suggest that aberrant multisensory processing is not necessarily linked to aberrant multisensory perception. In summary, our behavioral data did not reveal alterations in SIFI perception rates in SCZ. 


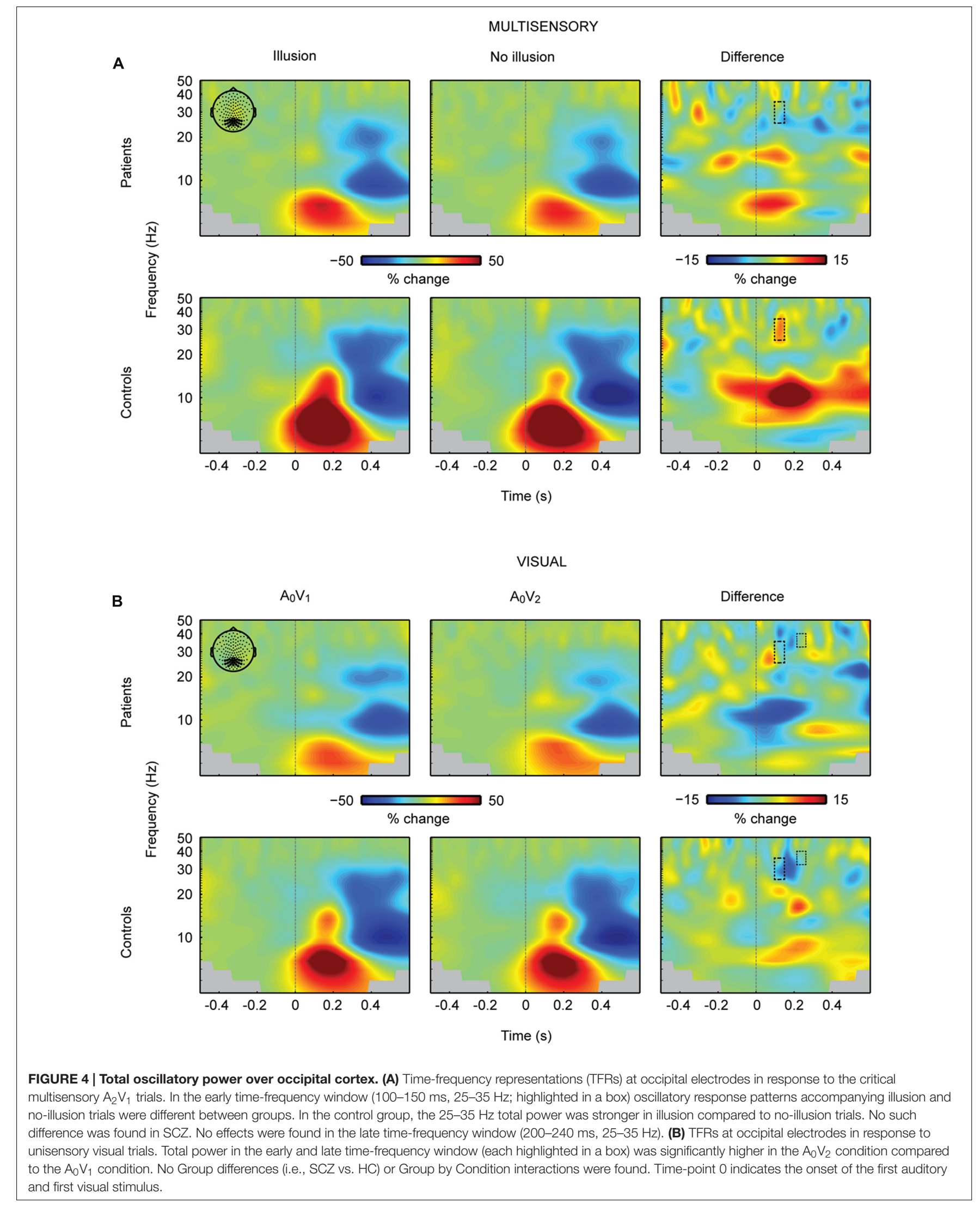




\section{Patients with Schizophrenia Show Diminished ERP Effects in the Sound-Induced Flash Illusion Paradigm}

Within HC, we observed larger ERP amplitudes around $140 \mathrm{~ms}$ and around $350 \mathrm{~ms}$ to illusion compared to no-illusion multisensory trials. This observation is comparable with previous findings in healthy individuals (Mishra et al., 2007; Keil A. et al., 2014). In the current study, HC compared to SCZ showed larger ERP amplitudes, irrespective of whether the critical SIFI trials were perceived as illusion or no-illusion. This indicates general group differences in the processing of multisensory stimuli. The ERP amplitudes around 140 ms were larger in HC compared to SCZ. Reduced EEG data quality in patients could have contributed to the reduced amplitudes. However, reduced ERP amplitudes in SCZ have been also found in numerous previous studies (e.g., Stone et al., 2011; Onitsuka et al., 2013; Stekelenburg et al., 2013). Furthermore, the number of rejected EEG trials in the analysis did not differ between groups. After the artifact rejection, there were on average 960 trials in the SCZ group and 953 trials in the control group. Therefore, we propose that the reduced ERP amplitudes in SCZ reflect processing alterations in patients and are not due to group differences in the EEG data quality. Taken together, reduced amplitudes in SCZ compared to HC following multisensory stimulation are found in early ERP components.

Importantly, we observed differences in multisensory integration effects, i.e., illusion vs. no-illusion trials, between groups. The integration effects, which were found around $135 \mathrm{~ms}$ after the onset of the first auditory and visual stimulus, were more robust in HC compared to SCZ (Figure 2). In unisensory processing, aberrant neural processing in SCZ often coincides with altered perception (Senkowski and Gallinat, 2015; Uhlhaas and Singer, 2015). Therefore, we expected that differences in multisensory processing in the SIFI would also affect the perceptual outcome. However, this was not the case. Recently, Stekelenburg et al. (2013) and Roa Romero et al. (2016b) also reported group differences in multisensory ERPs without showing effects in behavioral data. In an interesting framework on multisensory processing, Bizley et al. (2016) suggested different stages of multisensory integration, in which the initial integrative processing can occur, at least partially, independent of later perception-related processing. In line with this proposal, the initial processing and integration could be disturbed in the SIFI trials in SCZ, whereas the later perceptual stages might be still intact. Thus, there could be later mechanisms in the perception of multisensory stimuli in SCZ that compensate for earlier alterations in sensory processing by recruiting additional brain networks or additional regions within the same network (Rentrop et al., 2011). However, the assumption that compensatory mechanisms might support multisensory perception in SCZ requires further empirical testing. For instance, transcranial magnetic stimulation (TMS) could be applied at early and late time intervals following the presentation of SIFI trials. If there were compensatory mechanisms in SCZ then TMS applied specifically at late intervals should affect perception in patients. In sum, our study revealed altered processing of multisensory stimuli in SCZ. However, this did not affect the SIFI perception rate.

To better understand the observation of reduced multisensory processing in SCZ, the ERP findings in the critical multisensory SIFI trials can be compared to the findings in unisensory trials. Across groups, the ERP amplitudes of unisensory visual stimuli around $215 \mathrm{~ms}$ were larger in trials with two visual inputs compared to trials with one visual input. This effect was found in both groups, which might indicate that unisensory processing differences between 1 and 2 visual stimuli are comparable between SCZ and $\mathrm{HC}$ and that aberrant processing predominantly occurs in the processing of multisensory stimuli. Previous studies also found evidence for alterations in multisensory processing, but not in unisensory processing in SCZ (Ross et al., 2007). For example, Ross et al. (2007) found specific deficits in the ability of SCZ to integrate visual and auditory speech. SCZ experienced less benefit from visual articulation when they tried to comprehend speech under noisy environmental conditions, whereas their performance in unisensory auditory speech perception remained fully intact. This could imply that sensory processing in SCZ is particularly impaired when stimuli are presented in a multisensory way. The integration process of multisensory information might be more complex than the processing of information that is presented to only one sense.

\section{Schizophrenia Patients Lack an Enhancement of 25-35 Hz Power to Audiovisual Illusion Trials}

In the critical multisensory trials, HC displayed higher 25$35 \mathrm{~Hz}$ total power to illusion compared to no-illusion trials in the time window of 100 to $150 \mathrm{~ms}$ after stimulus onset. The observation of enhanced occipital power in healthy individuals replicates the previous finding by Mishra et al. (2007). Interestingly, the current study revealed that SCZ displayed no power differences at $25-35 \mathrm{~Hz}$ between illusion compared to no-illusion trials. Specifically, our study showed that patients lack an enhancement of neural oscillations over the occipital cortex to illusion trials. Interestingly, our data did not show general group differences for the $25-35 \mathrm{~Hz}$ responses to multisensory and unisensory visual or unisensory auditory stimuli. It might be that the absence of significant main effects between groups relates to a lack of statistical power. Indeed, previous studies have reported diminished evoked beta band $(13-30 \mathrm{~Hz})$ and gamma band $(>30 \mathrm{~Hz})$ oscillations in SCZ (Gallinat et al., 2004; Leicht et al., 2010, 2015; Lenz et al., 2011; Keil et al., 2016). For example, Leicht et al. (2010) investigated auditory evoked gamma band oscillations in SCZ and found a significant reduction of power in SCZ compared to $\mathrm{HC}$ around $50 \mathrm{~ms}$ after stimulus presentation. Keil et al. (2016) recently replicated this effect. Another study by Lenz et al. (2011) examined evoked gamma band responses in SCZ during an auditory oddball-paradigm and reported diminished responses to standard and deviant stimuli in SCZ around 40 to $90 \mathrm{~ms}$ after stimulus onset. These studies support our finding of diminished $25-35 \mathrm{~Hz}$ oscillations to 
illusory multisensory SIFI trials in SCZ. Our findings might indicate an early binding deficit in SCZ (Singer and Gray, 1995; Engel et al., 2001). Taken together with previous findings, our study suggests that altered $25-35 \mathrm{~Hz}$ oscillatory responses reflect aberrant multisensory processing in SCZ.

\section{CONCLUSION}

In this study, we compared multisensory processing of the SIFI paradigm between HC and SCZ. We found similar multisensory illusion rates between groups. Importantly, our analysis of ERP data revealed altered multisensory integration effects in SCZ compared to HC. Our findings are in agreement with recent ERP studies showing aberrant multisensory processing in SCZ. Moreover, our study complements recent reports of altered multisensory integration in the McGurk illusion, suggesting processing differences between SCZ and $\mathrm{HC}$ in various prominent multisensory illusion paradigms. Surprisingly, while differences in neural response patterns are relatively consistently observed, a number of studies revealed no alterations in behavior. Hence, further studies are required to uncover the precise associations between altered multisensory processing and behavioral as well as perceptual outcome in SCZ. Our analysis of neural oscillations revealed reduced occipital 25$35 \mathrm{~Hz}$ responses to illusory multisensory SIFI trials in SCZ. Thus far, neural oscillations during multisensory processing have been rarely investigated in SCZ. Previously, various studies have reported altered neural oscillations during unisensory processing in this patient group. Therefore, our observation of altered neural oscillations during multisensory processing provides further support for the notion that dysfunctional neural oscillations signify aberrant integrative sensory processing in SCZ.

\section{REFERENCES}

Andreasen, N. C. (2000). Schizophrenia: the fundamental questions. Brain Res. Brain Res. Rev. 31, 106-112. doi: 10.1016/S0165-0173(99)00027-2

Balz, J., Keil, J., Roa Romero, Y., Mekle, R., Schubert, F., Aydin, S., et al. (2016). GABA concentration in superior temporal sulcus predicts gamma power and perception in the sound-induced flash illusion. Neuroimage 125, 724-730. doi: 10.1016/j.neuroimage.2015.10.087

Bhattacharya, J., Shams, L., and Shimojo, S. (2002). Sound-induced illusory flash perception: role of gamma band responses. Neuroreport 13, 1727-1730. doi: 10.1097/00001756-200210070-00007

Bizley, J. K., Maddox, R. K., and Lee, A. K. C. (2016). Defining auditory-visual objects: behavioral tests and physiological mechanisms. Trends Neurosci. 39, 74-85. doi: 10.1016/j.tins.2015.12.007

Busse, L., and Woldorff, M. G. (2003). The ERP omitted stimulus response to "no-stim" events and its implications for fast-rate event-related fMRI designs. Neuroimage 18, 856-864. doi: 10.1016/S1053-8119(03)00012-0

Capa, R. L., Duval, C. Z., Blaison, D., and Giersch, A. (2014). Patients with schizophrenia selectively impaired in temporal order judgments. Schizophr. Res. 156, 51-55. doi: 10.1016/j.schres.2014.04.001

Chen, Y., Norton, D., and Stromeyer, C. III (2014). Prolonged temporal interaction for peripheral visual processing in schizophrenia: evidence from a three-flash illusion. Schizophr. Res. 156, 190-196. doi: 10.1016/j.schres.2014.04.018

de Boer-Schellekens, L., Stekelenburg, J. J., Maes, J. P., van Gool, A. R., and Vroomen, J. (2014). Sound improves diminished visual temporal sensitivity in schizophrenia. Acta Psychol. 147, 136-142. doi: 10.1016/j.actpsy.2013.06.013

\section{AUTHOR CONTRIBUTIONS}

JB conceived the experiment, recorded data, analyzed data, and wrote the manuscript. YR conceived the experiment, recorded data, and wrote the manuscript. JK conceived the experiment, analyzed data, and wrote the manuscript. MK analyzed data and wrote the manuscript. MN discussed the data and wrote the manuscript. JG conceived the experiment and wrote the manuscript. DS conceived the experiment, discussed data, and wrote the manuscript.

\section{FUNDING}

This work was supported by grants from the European Union (ERC-2010-StG-20091209 to DS) and the German Research Foundation (GA707/6-1 to JG, KE1828/2-1 to JK, and SE1859/41 to DS).

\section{ACKNOWLEDGMENTS}

The hand icon and speaker symbol in Figure $\mathbf{1}$ were made by Freepik and IconWorks, respectively, from flaticon.com. We would like to thank Tobias Bernklau, Melissa Henjes, Markus Koch and Paulina Schulz for their assistance in data collection, and Joseph Wooldridge for proofreading the manuscript.

\section{SUPPLEMENTARY MATERIAL}

The Supplementary Material for this article can be found online at: http://journal.frontiersin.org/article/10.3389/fpsyg. 2016.01896/full\#supplementary-material

Delorme, A., and Makeig, S. (2004). EEGLAB: an open source toolbox for analysis of single-trial EEG dynamics including independent component analysis. J. Neurosci. Methods 134, 9-21. doi: 10.1016/j.jneumeth.2003.10.009

Engel, A. K., Fries, P., and Singer, W. (2001). Dynamic predictions: oscillations and synchrony in top-down processing. Nat. Rev. Neurosci. 2, 704-716. doi: $10.1038 / 35094565$

Foucher, J. R., Lacambre, M., Pham, B. T., Giersch, A., and Elliott, M. A. (2007). Low time resolution in schizophrenia Lengthened windows of simultaneity for visual, auditory and bimodal stimuli. Schizophr. Res. 97, 118-127. doi: 10.1016/ j.schres.2007.08.013

Freedman, R., Waldo, M., Bickford-Wimer, P., and Nagamoto, H. (1991). Elementary neuronal dysfunctions in schizophrenia. Schizophr. Res. 4, 233-243. doi: 10.1016/0920-9964(91)90035-P

Gallinat, J., Winterer, G., Herrmann, C. S., and Senkowski, D. (2004). Reduced oscillatory gamma-band responses in unmedicated schizophrenic patients indicate impaired frontal network processing. Clin. Neurophysiol. 115, 18631874. doi: 10.1016/j.clinph.2004.03.013

Gardner, D. M., Murphy, A. L., O’Donnell, H. O., Centorrino, F., and Baldessarini, R. J. (2010). International consensus study of antipsychotic dosing. Am. J. Psychiatry 167, 686-693. doi: 10.1176/appi.ajp.2009.09060802

Giersch, A., Lalanne, L., van Assche, M., and Elliott, M. A. (2013). On disturbed time continuity in schizophrenia: an elementary impairment in visual perception? Front. Psychol. 4:281. doi: 10.3389/fpsyg.2013.00281

Gross, J. (2014). Analytical methods and experimental approaches for electrophysiological studies of brain oscillations. J. Neurosci. Methods 228, 57-66. doi: 10.1016/j.jneumeth.2014.03.007 
Gross, J., Baillet, S., Barnes, G. R., Henson, R. N., Hillebrand, A., Jensen, O., et al. (2013). Good practice for conducting and reporting MEG research. Neuroimage 65, 349-363. doi: 10.1016/j.neuroimage.2012.10.001

Javitt, D. C., and Freedman, R. (2015). Sensory processing dysfunction in the personal experience and neuronal machinery of schizophrenia. Am. J. Psychiatry 172, 17-31. doi: 10.1176/appi.ajp.2014.13121691

Kay, S. R., Fiszbein, A., and Opler, L. A. (1987). The positive and negative syndrome scale (PANSS) for schizophrenia. Schizophr. Bull. 13, 261-276. doi: 10.1093/ schbul/13.2.261

Keefe, R. S. E., Goldberg, T. E., Harvey, P. D., Gold, J. M., Poe, M. P., and Coughenour, L. (2004). The brief assessment of cognition in schizophrenia: reliability, sensitivity, and comparison with a standard neurocognitive battery. Schizophr. Res. 68, 283-297. doi: 10.1016/j.schres.2003.09.011

Keil, A., Debener, S., Gratton, G., Junghöfer, M., Kappenman, E. S., Luck, S. J., et al. (2014). Committee report: publication guidelines and recommendations for studies using electroencephalography and magnetoencephalography. Psychophysiology 51, 1-21. doi: 10.1111/psyp.12147

Keil, J., Müller, N., Hartmann, T., and Weisz, N. (2014). Prestimulus beta power and phase synchrony influence the sound-induced flash illusion. Cereb. cortex 24, 1278-1288. doi: 10.1093/cercor/bhs409

Keil, J., Roa Romero, Y., Balz, J., Henjes, M., and Senkowski, D. (2016). Positive and negative symptoms in schizophrenia relate to distinct oscillatory signatures of sensory gating. Front. Hum. Neurosci. 10:104. doi: 10.3389/fnhum.2016.00104

Lee, K.-H., Williams, L. M., Breakspear, M., and Gordon, E. (2003). Synchronous gamma activity: a review and contribution to an integrative neuroscience model of schizophrenia. Brain Res. Brain Res. Rev. 41, 57-78. doi: 10.1016/S01650173(02)00220-5

Lee, T.-W., Girolami, M., and Sejnowski, T. J. (1999). Independent component analysis using an extended infomax algorithm for mixed subgaussian and supergaussian sources. Neural Comput. 11, 417-441. doi: 10.1162/ 089976699300016719

Leicht, G., Andreou, C., Polomac, N., Lanig, C., Schöttle, D., Lambert, M. et al. (2015). Reduced auditory evoked gamma band response and cognitive processing deficits in first episode schizophrenia. World J. Biol. Psychiatry 16, 387-397. doi: 10.3109/15622975.2015.1017605

Leicht, G., Kirsch, V., Giegling, I., Karch, S., Hantschk, I., Möller, H.-J., et al. (2010). Reduced early auditory evoked gamma-band response in patients with schizophrenia. Biol. Psychiatry 67, 224-231. doi: 10.1016/j.biopsych.2009.07.033

Lenz, D., Fischer, S., Schadow, J., Bogerts, B., and Herrmann, C. S. (2011). Altered evoked gamma-band responses as a neurophysiological marker of schizophrenia? Int. J. Psychophysiol. 79, 25-31. doi: 10.1016/j.ijpsycho.2010.08. 002

Lisman, J. (2012). Excitation, inhibition, local oscillations, or large-scale loops: what causes the symptoms of schizophrenia? Curr. Opin. Neurobiol. 22, 537-544. doi: 10.1016/j.conb.2011.10.018

Maris, E. (2012). Statistical testing in electrophysiological studies. Psychophysiology 49, 549-565. doi: 10.1111/j.1469-8986.2011.01320.x

Maris, E., and Oostenveld, R. (2007). Nonparametric statistical testing of EEG- and MEG-data. J. Neurosci. Methods 164, 177-190. doi: 10.1016/j.jneumeth.2007.03. 024

Martin, B., Giersch, A., Huron, C., and van Wassenhove, V. (2013). Temporal event structure and timing in schizophrenia: preserved binding in a longer "now". Neuropsychologia 51, 358-371. doi: 10.1016/j.neuropsychologia.2012. 07.002

McGhie, A., and Chapman, J. (1961). Disorders of attention and perception in early schizophrenia. Br. J. Med. Psychol. 34, 103-116. doi: 10.1111/j.2044-8341.1961. tb00936.x

Mishra, J., Martinez, A., and Hillyard, S. A. (2008). Cortical processes underlying sound-induced flash fusion. Brain Res. 1242, 102-115. doi: 10.1016/j.brainres. 2008.05.023

Mishra, J., Martínez, A., and Hillyard, S. A. (2010). Effect of attention on early cortical processes associated with the sound-induced extra flash illusion. J. Cogn. Neurosci. 22, 1714-1729. doi: 10.1162/jocn.2009.21295

Mishra, J., Martinez, A., Sejnowski, T. J., and Hillyard, S. A. (2007). Early crossmodal interactions in auditory and visual cortex underlie a sound-induced visual illusion. J. Neurosci. 27, 4120-4131. doi: 10.1523/JNEUROSCI.4912-06. 2007
Norton, D., Ongur, D., Stromeyer, C. III, and Chen, Y. (2008). Altered “three-flash" illusion in response to two light pulses in schizophrenia. Schizophr. Res. 103, 275-282. doi: 10.1016/j.schres.2008.03.002

Onitsuka, T., Oribe, N., Nakamura, I., and Kanba, S. (2013). Review of neurophysiological findings in patients with schizophrenia. Psychiatry Clin. Neurosci. 67, 461-470. doi: 10.1111/pcn.12090

Oostenveld, R., Fries, P., Maris, E., and Schoffelen, J.-M. (2011). FieldTrip: open source software for advanced analysis of MEG, EEG, and invasive electrophysiological data. Comput. Intell. Neurosci. 2011:156869. doi: 10.1155/ 2011/156869

Pearl, D., Yodashkin-Porat, D., Katz, N., Valevski, A., Aizenberg, D., Sigler, M., et al. (2009). Differences in audiovisual integration, as measured by McGurk phenomenon, among adult and adolescent patients with schizophrenia and agematched healthy control groups. Compr. Psychiatry 50, 186-192. doi: 10.1016/j. comppsych.2008.06.004

Pittman-Polletta, B. R., Kocsis, B., Vijayan, S., Whittington, M. A., and Kopell, N. J. (2015). Brain rhythms connect impaired inhibition to altered cognition in schizophrenia. Biol. Psychiatry 77, 1020-1030. doi: 10.1016/j.biopsych.2015. 02.005

Rentrop, M., Roth, A., Rodewald, K., Simon, J., Metzler, S., Walther, S., et al. (2011). Temporal variability and spatial diffusion of the N2 event-related potential in high-functioning patients with schizophrenia. Schizophr. Res. 131, 206-213. doi: 10.1016/j.schres.2011.06.020

Roa Romero, Y., Keil, J., Balz, J., Niedeggen, M., Gallinat, J., and Senkowski, D. (2016a). Alpha-band oscillations reflect altered multisensory processing of the McGurk illusion in schizophrenia. Front. Hum. Neurosci. 10:41. doi: 10.3389/ fnhum.2016.00041

Roa Romero, Y., Keil, J., Balz, J., Gallinat, J., Senkowski, D. (2016b). Reduced frontal theta oscillations indicate altered crossmodal prediction error processing in schizophrenia. J. Neurophysiol. 116, 1396-1407. doi: 10.1152/jn. 00096.2016

Ross, L. A., Saint-Amour, D., Leavitt, V. M., Molholm, S., Javitt, D. C., and Foxe, J. J. (2007). Impaired multisensory processing in schizophrenia: deficits in the visual enhancement of speech comprehension under noisy environmental conditions. Schizophr. Res. 97, 173-183. doi: 10.1016/j.schres.2007.08.008

Senkowski, D., and Gallinat, J. (2015). Dysfunctional prefrontal gammaband oscillations reflect working memory and other cognitive deficits in schizophrenia. Biol. Psychiatry 77, 1010-1019. doi: 10.1016/j.biopsych.2015.02. 034

Senkowski, D., Saint-Amour, D., Kelly, S. P., and Foxe, J. J. (2007). Multisensory processing of naturalistic objects in motion: a high-density electrical mapping and source estimation study. Neuroimage 36, 877-888. doi: 10.1016/j. neuroimage.2007.01.053

Shakow, D. (1963). Psychological deficit in schizophrenia. Behav. Sci. 8, 275-305. doi: 10.1002/bs.3830080402

Shams, L., Kamitani, Y., and Shimojo, S. (2000). Illusions. What you see is what you hear. Nature 408: 788. doi: 10.1038/35048669

Singer, W., and Gray, C. M. (1995). Visual feature integration and the temporal correlation hypothesis. Annu. Rev. Neurosci. 18, 555-586. doi: 10.1146/annurev. ne.18.030195.003011

Spencer, K. M., Salisbury, D. F., Shenton, M. E., and Mccarley, R. W. (2008). Gamma-band auditory steady-state responses are impaired in first episode psychosis. Biol. Psychiatry 64, 369-375. doi: 10.1016/j.biopsych.2008. 02.021

Stekelenburg, J. J., Maes, J. P., Van Gool, A. R., Sitskoorn, M., and Vroomen, J. (2013). Deficient multisensory integration in schizophrenia: an event-related potential study. Schizophr. Res. 147, 253-261. doi: 10.1016/j.schres.2013.04.038

Stevenson, R. A., Park, S., Cochran, C., McIntosh, L. G., Noel, J. P., Barense, M. D., et al. (2016). The associations between multisensory temporal processing and symptoms of schizophrenia. Schizophr. Res. doi: 10.1016/j.schres.2016.09.035 [Epub ahead of print].

Stone, D. B., Coffman, B. A., Bustillo, J. R., Aine, C. J., and Stephen, J. M. (2014). Multisensory stimuli elicit altered oscillatory brain responses at gamma frequencies in patients with schizophrenia. Front. Hum. Neurosci. 8:788. doi: 10.3389/fnhum.2014.00788

Stone, D. B., Urrea, L. J., Aine, C. J., Bustillo, J. R., Clark, V. P., and Stephen, J. M. (2011). Unisensory processing and multisensory integration in schizophrenia: 
a high-density electrical mapping study. Neuropsychologia 49, 3178-3187. doi: 10.1016/j.neuropsychologia.2011.07.017

Surguladze, S. A., Calvert, G. A., Brammer, M. J., Campbell, R., Bullmore, E. T., Giampietro, V., et al. (2001). Audio-visual speech perception in schizophrenia: an fMRI study. Psychiatry Res. 106, 1-14. doi: 10.1016/S0925-4927(00)00081-0

Tseng, H., Bossong, M. G., Modinos, G., Chen, K., McGuire, P., and Allen, P. (2015). A systematic review of multisensory cognitive-affective integration in schizophrenia. Neurosci. Biobehav. Rev. 55, 444-452. doi: 10.1016/j.neubiorev. 2015.04.019

Uhlhaas, P. J., Linden, D. E. J., Singer, W., Haenschel, C., Lindner, M., Maurer, K., et al. (2006). Dysfunctional long-range coordination of neural activity during Gestalt perception in schizophrenia. J. Neurosci. 26, 8168-8175. doi: 10.1523/ JNEUROSCI.2002-06.2006

Uhlhaas, P. J., and Singer, W. (2015). Oscillations and neuronal dynamics in schizophrenia: the search for basic symptoms and translational opportunities. Biol. Psychiatry 77, 1001-1009. doi: 10.1016/j.biopsych.2014.11.019

van Wassenhove, V., Grant, K. W., and Poeppel, D. (2005). Visual speech speeds up the neural processing of auditory speech. Proc. Natl. Acad. Sci. U.S.A. 102, 1181-1186. doi: 10.1073/pnas.0408949102
Vlcek, P., Bob, P., and Raboch, J. (2014). Sensory disturbances, inhibitory deficits, and the P50 wave in schizophrenia. Neuropsychiatr. Dis. Treat. 10, 1309-1315. doi: $10.2147 /$ NDT.S64219

Wynn, J. K., Jahshan, C., and Green, M. F. (2014). Multisensory integration in schizophrenia: a behavioural and event-related potential study. Cogn. Neuropsychiatry 19, 319-336. doi: 10.1080/13546805.2013. 866892

Conflict of Interest Statement: The authors declare that the research was conducted in the absence of any commercial or financial relationships that could be construed as a potential conflict of interest.

Copyright (c) 2016 Balz, Roa Romero, Keil, Krebber, Niedeggen, Gallinat and Senkowski. This is an open-access article distributed under the terms of the Creative Commons Attribution License (CC BY). The use, distribution or reproduction in other forums is permitted, provided the original author(s) or licensor are credited and that the original publication in this journal is cited, in accordance with accepted academic practice. No use, distribution or reproduction is permitted which does not comply with these terms. 